\title{
On the Performances of Density Functionals for Open Shell First-row Transition Metal Compounds
}

Yumiao Ma

BSJ Institute, Haidian, Beijing, 100084

ymma@bsj-institute.top

\begin{abstract}
The selection of density functional is the key to obtain useful results in a computational work. Due to their complexity in terms of electronic structures, open-shell first-row transition metal complexes are difficult to be correctly described by most functionals. In this work, totally 19 reactions involving $\mathrm{V}, \mathrm{Cr}, \mathrm{Mn}, \mathrm{Fe}, \mathrm{Co}$, Ni complexes, either monometallic or bimetallic, were used as testing set for 18 functionals ranging from generalized gradient approximation (GGA) to doublyhybrid functionals, with experimental electron affinities and ligand association energies as standard. It is shown that for monometallic complexes PBE0-D3BJ and B3LYP-D3BJ perform the best, whereas MN15 and MN15L are the optimal functionals for bimetallic compounds. On the other hand, the accuracy of DLPNO-CCSD(T) is not significantly better than the best-performing functionals, and the use of doubly-hybrid functionals is risky.
\end{abstract}

\section{Introduction}

Density functional theory (DFT) ${ }^{1}$ has been the most widely used computational method nowadays, due to its balanced accuracy and time consuming. A huge number of density functionals, designed by diverse methodologies and with different approximations, have been developed over the past decades. At present there is no functional that gives perfect accuracy in all cases, and thus it is crucial for any computational scientist to choose a proper functional before starting a computational research on chemical reactions. While accurate evaluation of energetics is always important, accuracies for open shell (especially the first row transition metals, which usually have small gaps between spin states and are prior to adopt high spin ground states) transition metals are in higher and higher demand in the recent years with the rapid development of cheap transition metal catalysis. On the other hand, these systems usually feature high multi-reference characters and complex electronic structures, and it could be expected that they are much more challenging for most functionals.

In order to obtain general knowledge about the performances of density functionals, a vast number of testing sets have been proposed, and vast benchmark studies were performed. In 2006, for example, Jan Martin tested the performance of several functionals in Pd-catalyzed C-X bond activation and Heck reactions ${ }^{2}$, with coupled cluster results as standard. The famous WCCR10 testing set ${ }^{3}$, involving the thermodynamics of 10 transition metal reactions, was suggested by Markus Reiher in 2014, and contains 8 reactions involving low spin Pt, Pd, Ag, Au complexes, and 2 reactions with close shell $\mathrm{Cu}(\mathrm{I})$. In 2018, Stefan Grimme reported a comprehensive benchmark study on the performances of 41 functionals on 41 close shell transition metal reactions (namely the MOR41 set) ${ }^{4}$. In contrast to the fact that various density functionals were thoroughly tested for close shell transition metal compounds, much less benchmark studies are focused on open shell first-row transition metal systems.

Actually there are some databases involving open shell systems, especially those established by Donald Truhlar for developing new functionals. In a study in 2005, a database containing the bond dissociation energies for $\mathrm{CoH}, \mathrm{CoOH}^{+}$, and other 20 molecules was suggested ${ }^{5}$, with 
experimental values as standard. Later on, several other databases involving simple high spin firstrow transition metal compounds were reported ${ }^{6-9}$, and finally compiled into the database 2015B used to develop the MN15 functional ${ }^{10}$. Besides Truhlar's work, Kasper Jensen and coworkers published their benchmark on the geometries and bond dissociation energies of diatomic compounds formed by first-row transition metals and several main group elements in $2007^{11}$. However, since these databases are used both in fitting and testing, it is questionable how these functionals perform in real situations with different nature from the testing sets. In this work, a benchmark study was performed based on a new testing set that was never accepted into any training databases to the best of the author's knowledge, and the performance of several popular functionals were compared.

\section{The Testing Set and Computational Methods}

The testing set in this work contains 16 adiabatic electron affinities, 1 vertical electron affinity, and 2 ligand association energies. All standard values were experimentally determined by photoelectron spectroscopy and related technologies. There are many reasons for choosing these data as the testing set. They were measured in gas phase, with a relatively small error bar. The absolute values of the energetics are similar to general chemical reactions, and since the first-row transition metals are prior to undergo single electron transfer in actual reactions, it is proposed that the behavior of functionals on these examples should provide some insights for real situations, although most of the molecules tested are small. The full list of the testing set is shown in Table 1.

Table 1. The reactions included in the testing set

\begin{tabular}{|c|c|c|c|c|}
\hline $\begin{array}{l}\text { Reaction } \\
\text { Number }\end{array}$ & Reaction & Value $(\mathrm{kcal} / \mathrm{mol})$ & $\begin{array}{ll}\text { Error } & \text { bar } \\
(\mathrm{kcal} / \mathrm{mol}) & \end{array}$ & Reference \\
\hline \multicolumn{5}{|c|}{12 adiabatic electron affinities for monometallic compounds } \\
\hline 1 & $\mathrm{FeBz}->\mathrm{FeBz}^{-}$ & -10.6 & 2.3 & 12 \\
\hline 2 & $\mathrm{VBz}->\mathrm{VBz}^{-}$ & -14.3 & 1.6 & 13 \\
\hline 3 & $\begin{array}{ll}\mathrm{Co}(\mathrm{CO})_{2} \mathrm{NO} & -> \\
\mathrm{Co}(\mathrm{CO})_{2} \mathrm{NO}^{-} & \\
\end{array}$ & -39.90 & 0.69 & 14 \\
\hline 4 & $\mathrm{CoH}->\mathrm{CoH}^{-}$ & -15.47 & 0.23 & 15 \\
\hline 5 & $\mathrm{NiH}->\mathrm{NiH}^{-}$ & -11.09 & 0.16 & 15 \\
\hline 6 & $\mathrm{CrH}->\mathrm{CrH}^{-}$ & -18.61 & 0.23 & 15 \\
\hline 7 & $\mathrm{FeH}->\mathrm{FeH}^{-}$ & -21.54 & 0.25 & 16 \\
\hline 8 & $\mathrm{MnH}->\mathrm{MnH}^{-}$ & -20.04 & 0.23 & 16 \\
\hline 9 & $\mathrm{MnH}_{2}->\mathrm{MnH}_{2}^{-}$ & -10.24 & 0.37 & 17 \\
\hline 10 & $\mathrm{FeH}_{2}->\mathrm{FeH}_{2}^{-}$ & -24.19 & 0.32 & 17 \\
\hline 11 & $\mathrm{FeO}->\mathrm{FeO}^{-}$ & -34.4 & 2.3 & 18 \\
\hline 12 & $\mathrm{FeS}->\mathrm{FeS}^{-}$ & -40.59 & 0.05 & 19 \\
\hline \multicolumn{5}{|c|}{1 vertical electron affinity } \\
\hline 13 & $\begin{array}{ll}\mathrm{Cu}\left(\mathrm{H}_{2} \mathrm{O}\right)_{2} \\
\mathrm{Cu}\left(\mathrm{H}_{2} \mathrm{O}\right)_{2}^{-}\end{array}$ & -46.12 & 0.69 & 20 \\
\hline \multicolumn{5}{|c|}{2 ligand association energies } \\
\hline 14 & $\begin{array}{l}\mathrm{FeCS}_{2}^{+}+\mathrm{CS}_{2}-> \\
\mathrm{Fe}\left(\mathrm{CS}_{2}\right)_{2}^{+}\end{array}$ & -45.0 & 1.4 & 21 \\
\hline 15 & $\mathrm{~V}\left(\mathrm{C}_{2} \mathrm{H}_{4}\right)^{+}+\mathrm{C}_{2} \mathrm{H}_{4}->$ & -29.6 & 1.9 & 22 \\
\hline
\end{tabular}




\begin{tabular}{|l|l|l|l|l|}
\hline \multicolumn{2}{|l|}{$\mathrm{V}_{\left(\mathrm{C}_{2} \mathrm{H}_{4}\right)_{2}{ }^{+}}$} & \multicolumn{2}{l|}{} \\
\hline \multicolumn{2}{|l|}{ adiabatic electron affinities for bimetallic compounds } \\
\hline 16 & $\mathrm{Cr}_{2} \mathrm{H}->\mathrm{Cr}_{2} \mathrm{H}^{-}$ & -33.99 & 0.11 & 23 \\
\hline 17 & $\mathrm{Fe}_{2} \mathrm{Bz}->\mathrm{Fe}_{2} \mathrm{Bz}^{-}$ & -26.5 & 2.3 & 12 \\
\hline 18 & $\mathrm{Ni}_{2} \mathrm{Bz}->\mathrm{Ni}_{2} \mathrm{Bz}^{-}$ & -23.1 & 2.3 & 24 \\
\hline 19 & $\mathrm{Fe}_{2} \mathrm{O}->\mathrm{Fe}_{2} \mathrm{O}^{-}$ & -37.59 & 0.92 & 25 \\
\hline
\end{tabular}

$* \mathrm{Bz}=\mathrm{C}_{6} \mathrm{H}_{6}$

All calculations were performed with the ORCA 4.2.0 program $^{26,27}$. Totally 19 functionals were tested: PBE-D3BJ ${ }^{28,}$ 29, BP86-D3BJ ${ }^{30,} 31$, TPSS-D3BJ ${ }^{32}$, M06L-D3 ${ }^{33}$, MN15L ${ }^{34}$, TPSSh-

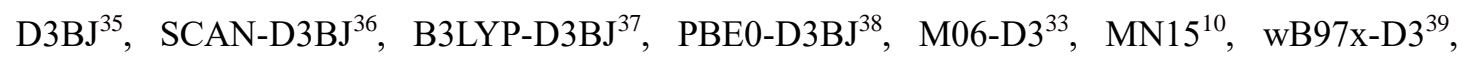
${ }_{\text {wB97M-V }}^{40}, \quad$ wB97X-V ${ }^{41}, \quad$ B2PLYP-D3BJ ${ }^{42}, \quad$ B2GPLYP-D3BJ ${ }^{43}$, DSD-PBEP86-D3BJ ${ }^{44}$, ${ }_{\text {wB2PGLYP }}^{45}$, PWPB95-D3BJ ${ }^{46}$. In contrast to most benchmark studies that geometries were optimized at a fixed level, in this work all geometries were optimized with the corresponding functional in combination with the def2-TZVP basis set ${ }^{47}$, unless especially noted. For example, the results for PBE-D3BJ were based on the geometry optimized at PBE-D3BJ/def2-TZVP level, and those for PBE0-D3BJ were based on geometry optimized at PBE0-D3BJ/def2-TZVP level. The RI and RIJCOSX techniques ${ }^{48}$ were used to accelerate DFT calculations (but not the CCSD(T) calculations), with the "GridX6 NoFinalGridX" keywords. The auxiliary basis set was chosen as def2-TZVP/C, aug-cc-pVTZ/C, aug-cc-pVQZ/C for the def2-TZVP, aug-cc-pVTZ ${ }^{49}$, aug-cc$\mathrm{pVQZ}^{49}$ basis sets respectively, and the AutoAux feature in ORCA was used to generate auxiliary basis set for ma-def2-TZVP50. The keywords "TightSCF Grid6 NoFinalGrid" were always used. The stability of all wavefunctions was examined. All the possible spin states were examined for all compounds, and only results corresponding to ground states were shown below. For DLPNO$\operatorname{CCSD}(\mathrm{T})$ calculations, the VeryTightSCF and TightPNO keywords were used.

NOTE: For some compounds the ORCA program may give results that correspond to a stable wavefunction, but the energies are unreasonable. For these structures there are more than one stable wavefunctions. Thus it is crucial to repeat all calculations with various initial guess modes (the default method, reading results at other levels, reading results for compounds with other charge number, or even reading from results generated by other programs, if possible).

\section{Results and Discussions}

\section{Energetics}

All compounds involved in Table 1 were optimized with various functionals in combination with the def2-TZVP basis set. Then a single point energy calculation at aug-cc-pVTZ level was performed, and the so-obtained zero point energies (ZPEs) were used to compare the accuracy of functionals examined.

At the very first, the GGA, meta-GGA and hybrid functionals were examined, because their analytical gradients are available in ORCA. The errors are shown in Table 2.

Table 2. Errors, MSEs (mean signed errors), MUEs (mean unsigned errors), maximum errors, and STDEVs (standard deviation for signed errors) of several functionals on the testing set (in 
$\mathrm{kcal} / \mathrm{mol})$.

\begin{tabular}{|lllllllllllll}
\hline Reaction Number PBE & BP86 & TPSS & M06L & SCAN & MN15L & PBE0 & TPSSh & B3LYP & M06 & MN15 \\
\hline 1 & -2.47 & -5.73 & -0.38 & $-*$ & -3.51 & -5.86 & -1.74 & 0.41 & -0.17 & 1.94 & 2.42 \\
2 & -8.39 & -9.79 & -8.59 & $-*$ & -8.67 & -4.09 & -1.05 & -3.29 & 0.00 & 3.07 & 3.61 \\
\hline 3 & -3.87 & -6.40 & -2.57 & 1.32 & -4.62 & -2.34 & 0.13 & -0.94 & -0.87 & -0.86 & -3.71 \\
\hline 4 & -1.25 & -4.22 & -0.45 & -0.17 & -8.25 & -1.86 & -0.03 & 0.06 & 0.73 & -0.29 & 4.10 \\
\hline 5 & -9.43 & -11.87 & -4.30 & 1.47 & -4.46 & -8.23 & 1.07 & -0.79 & 0.31 & -3.37 & -1.31 \\
\hline 6 & 0.01 & -2.40 & -0.01 & 4.01 & 2.14 & -2.47 & -0.09 & 0.11 & 0.04 & -5.31 & 2.93 \\
\hline 7 & 1.35 & -4.21 & 1.80 & -4.94 & -2.33 & -8.58 & 1.97 & 0.01 & 3.94 & -9.17 & 1.40 \\
\hline 8 & -3.10 & -6.69 & -0.09 & -4.98 & 3.13 & -7.79 & 3.61 & 2.48 & -2.22 & -12.93 & -6.60 \\
\hline 9 & -10.84 & -13.29 & -6.84 & -1.83 & -2.74 & -3.41 & -0.07 & -2.89 & -5.74 & -10.93 & -6.52 \\
\hline 10 & -5.59 & -11.90 & -7.58 & -0.03 & -0.79 & -3.29 & 3.13 & -8.31 & 3.56 & -4.80 & 3.20 \\
\hline 11 & 3.66 & 1.12 & 6.69 & 3.96 & 5.52 & 1.04 & 4.50 & 7.04 & 2.25 & -4.93 & -1.43 \\
12 & 4.80 & 2.27 & 7.07 & 7.22 & 4.90 & -1.25 & 3.48 & 6.69 & 2.53 & -1.99 & 0.50 \\
\hline 13 & -4.26 & -6.65 & 0.35 & 1.36 & $-* *$ & -5.38 & 3.83 & 3.30 & 0.70 & -4.14 & -2.96 \\
\hline 14 & -1.74 & -3.10 & 1.12 & 3.36 & -0.12 & 1.01 & 6.20 & 4.26 & 7.91 & 0.76 & 4.93 \\
\hline 15 & -7.46 & -7.54 & -5.53 & -4.62 & -10.85 & 1.31 & -1.71 & -3.18 & -0.15 & -2.34 & -4.89 \\
\hline 16 & 10.75 & 7.17 & 12.53 & 5.30 & 11.15 & -6.46 & 8.51 & 9.93 & 6.75 & -4.08 & 1.33 \\
\hline 17 & -6.29 & -8.52 & -0.62 & 7.79 & -2.43 & 3.38 & 8.82 & 10.03 & 11.79 & 8.88 & 2.16 \\
18 & -3.56 & -8.29 & 8.74 & 11.77 & 2.36 & -1.36 & 9.08 & 4.36 & 4.52 & -0.69 & -5.79 \\
\hline 19 & 8.23 & 5.22 & 9.83 & 9.53 & 4.52 & -0.50 & 5.86 & 8.56 & 6.41 & 4.11 & 4.46 \\
\hline MSE & -2.08 & -4.99 & 0.59 & 2.38 & -0.84 & -2.96 & 2.92 & 1.99 & 2.23 & -2.48 & -0.11 \\
\hline MUE & 5.11 & 6.65 & 4.48 & 4.33 & 4.58 & 3.66 & 3.41 & 4.03 & 3.19 & 4.45 & 3.38 \\
\hline Max Error & 10.84 & 13.29 & 12.53 & 11.77 & 11.15 & 8.58 & 9.08 & 10.03 & 11.79 & 12.93 & 6.60 \\
\hline STDEV & 5.66 & 5.52 & 5.88 & 4.83 & 5.48 & 3.36 & 3.44 & 4.84 & 3.93 & 5.10 & 3.82 \\
\hline & & & & & & & & & &
\end{tabular}

*. Severe SCF convergence problem met using the aug-cc-pVTZ basis set.

**. The optimized geometry dissociated.

It is seen from Table 2 that the errors for all functionals are rather distributed, and there is no functional that performs well in all reactions. In terms of mean unsigned error (MUE), B3LYPD3BJ (MUE=3.19 kcal/mol) MN15 (3.38 kcal/mol), and PBE0-D3BJ (MUE=3.41 kcal/mol) are the optimal functionals for the whole testing set, and MN15L (MUE=3.66 kcal $/ \mathrm{mol}$ ) also performs well. All other functionals exhibit MUEs around 4-6 kcal $/ \mathrm{mol}$. The position on the Jacob's ladder is generally consistent with the overall performance, and PBE-D3BJ and BP86-D3BJ exhibit the largest MUE, despite they are widely used for transition metal complexes. The theoretically-derived SCAN-D3BJ functional performed moderately, and failed to give the correct configuration of $\mathrm{Cu}$ water complex. The two Minnesota functionals, M06-D3 and M06L-D3, also gave moderate errors.

Besides the MUEs, the standard deviation of signed errors (STDEV) were also used to judge the robustness of functionals. Among all the functionals shown in Table 2, PBE0-D3BJ and MN15L exhibits the smallest STDEVs, meaning that they have a narrower distribution of errors in all reactions. In the contrast, PBE-D3BJ, BP86-D3BJ, TPSS-D3BJ and SCAN-D3BJ exhibit the largest STDEVs.

It is noticed that the bimetallic compounds (16-19) are more difficult than others, and contribute most of the MUEs for PBE0-D3BJ and B3LYP-D3BJ, whereas the errors of the two functionals for the monometallic examples are generally small. The MUEs, MSEs, and STDEVs for monometallic and bimetallic examples were shown in Figure 1, and it is clear that PBE0-D3BJ and B3LYP-D3BJ are the best functionals for the monometallic testing sets, although they perform terribly for the bimetallic examples. It is easily understandable that the bimetallic compounds should exhibit more complex electronic structure, more multi-reference characters, and are more challenging for single-reference DFT theory. In this work, only MN15L (MUE=2.93 kcal $/ \mathrm{mol}$ ) and MN15 (MUE=3.43 kcal/mol) gave acceptable results for the entries 16-19. Serge Gorelsky noted in 2012 that hybrid functionals generally failed to describe metal-metal bonding ${ }^{51}$, and thus the MN15L functional is recommended for calculating compounds with metal-metal bonds. 


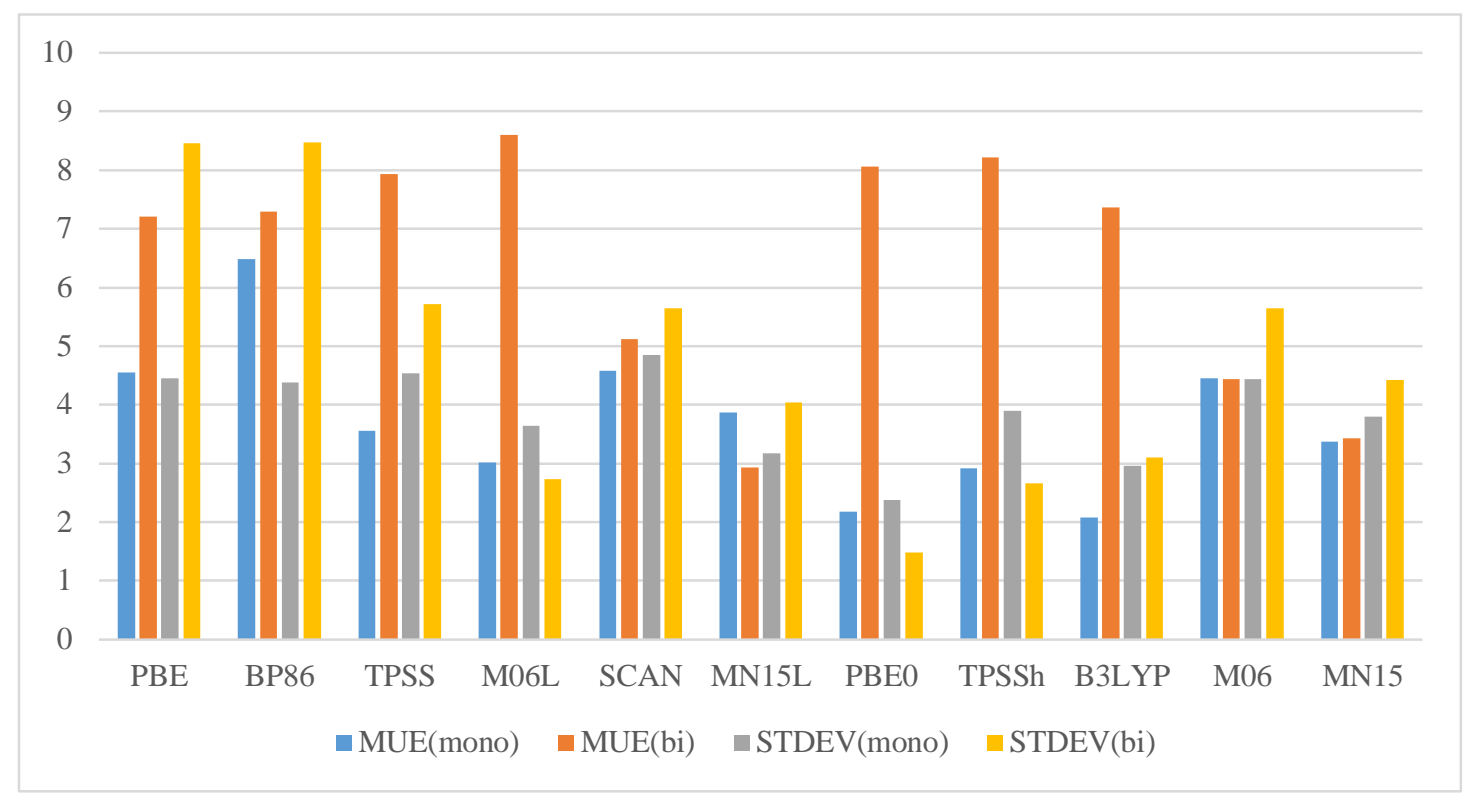

Figure 1. The MUEs and STDEVs for the examined functionals in monometallic and bimetallic cases.

\section{Geometries}

The geometries optimized with various functionals were compared with experimental values, together with DLPNO-CCSD(T) results (Table 3). Since there are no analytical gradients for both CCSD and CCSD(T), only linear molecules were examined, and the DLPNO-CCSD(T) results were obtained by rigid scanning. All the bond lengths listed are for the corresponding ground state, determined with each functional. The general trend that hybrid functionals outperform meta-GGAs, and the latter outperform GGAs, is again clear. The most impressive observation is that the SCAND3BJ functional gave much worse bond lengths compared to all other functionals, although its performance on energetics is moderate (Figure 1). The accuracy of DLPNO-CCSD(T) for these compounds is also moderate, and several functionals (B3LYP, M06, MN15L, PBE0, M06L) outperformance DLPNO-CCSD(T) with respect to geometry. It is in consistence with the general observation of large spin contaminant for UHF calculations. Also it is interesting that for $\mathrm{CoH}^{-}$, all functionals predicted a quartet ground state with quartet-doublet gaps ranging from $2 \mathrm{kcal} / \mathrm{mol}$ (for GGAs and meta-GGAs) to $20 \mathrm{kcal} / \mathrm{mol}$ (for hybrid functionals), whereas DLPNO-CCSD(T) afforded a doublet ground state and the quartet state dissociated spontaneously. The energetic errors of DLPNO-CCSD(T) was shown in Table 4, and is not better than the best-performing functionals in the Energetics part. In a summary, it should be concluded that singlet-reference DLPNO$\operatorname{CCSD}(\mathrm{T})$ cannot be considered as an absolute standard for open shell transition metal complexes.

Table 3. Errors in the bond length (in angstrom) in structures optimized by various functionals (with def2-TZVP basis set) and DLPNO-CCSD(T)/aug-cc-pVTZ level.

\begin{tabular}{llllllllllllll}
\hline & PBE & BP86 & TPSS & M06L & SCAN & MN15L & PBE0 & TPSSh & B3LYP & M06 & MN15 & CCSD(T) & Exp. \\
\hline $\mathrm{CoH}$ & -0.021 & -0.074 & -0.017 & -0.017 & 0.279 & 0.006 & -0.017 & -0.017 & -0.017 & -0.013 & -0.070 & -0.042 & 1.542 \\
$\mathrm{CoH}-$ & -0.017 & -0.013 & -0.018 & 0.011 & 0.079 & 0.083 & -0.023 & -0.020 & -0.008 & 0.000 & 0.021 & -0.069 & 1.670 \\
$\mathrm{CrH}$ & -0.002 & -0.003 & -0.001 & 0.016 & -0.120 & 0.003 & 0.008 & 0.003 & 0.008 & 0.017 & -0.024 & -0.045 & 1.655 \\
$\mathrm{CrH}-$ & -0.051 & -0.051 & -0.044 & -0.025 & -0.216 & -0.055 & -0.023 & -0.033 & -0.023 & -0.018 & -0.092 & -0.030 & 1.750 \\
$\mathrm{NiH}$ & 0.009 & 0.007 & 0.015 & 0.029 & 0.117 & 0.021 & 0.045 & 0.029 & 0.043 & 0.036 & 0.025 & 0.041 & 1.476 \\
$\mathrm{FeH}$ & -0.097 & -0.099 & -0.090 & -0.063 & 0.043 & -0.032 & -0.078 & -0.083 & -0.076 & -0.064 & -0.065 & -0.067 & 1.630 \\
$\mathrm{FeH}-$ & -0.090 & -0.087 & -0.090 & -0.091 & -0.112 & 0.002 & -0.087 & -0.090 & -0.076 & -0.068 & -0.055 & -0.040 & 1.790 \\
$\mathrm{MnH}$ & -0.004 & 0.000 & -0.003 & 0.031 & -0.078 & 0.051 & 0.006 & 0.001 & 0.023 & -0.002 & -0.006 & 0.005 & 1.731 \\
$\mathrm{MnH}-$ & -0.068 & -0.063 & -0.068 & -0.018 & -0.136 & 0.033 & -0.032 & -0.057 & -0.024 & -0.038 & -0.036 & -0.020 & 1.820 \\
$\mathrm{MUE}$ & 0.040 & 0.044 & 0.038 & 0.034 & 0.131 & 0.032 & 0.035 & 0.037 & 0.033 & 0.029 & 0.044 & 0.040 & \\
\hline
\end{tabular}


* The experimental value for $\mathrm{CrH}$ is from reference ${ }^{52}$.

Table 4. Energetic errors for DLPNO-CCSD(T)/aug-cc-pVTZ.

\begin{tabular}{|l|l|}
\hline Reaction Number & Error $(\mathrm{kcal} / \mathrm{mol})$ \\
\hline 4 & -2.21 \\
\hline 5 & -7.52 \\
\hline 6 & 2.56 \\
\hline 7 & -0.32 \\
\hline 9 & 2.18 \\
\hline 11 & 5.18 \\
\hline 12 & 7.44 \\
\hline
\end{tabular}

\section{Basis Set Convergence}

The convergence of MUEs with increased basis set size was investigated by comparing the results with single point energies obtained with def2-TZVP, ma-def2-TZVP, aug-cc-pVTZ basis set respectively, based on the def2-TZVP geometries. The larger 4-zeta basis set was not considered due to its time consuming and SCF convergence problems. Interestingly not all functionals exhibit consistent decrease of MUE with larger basis set. Inclusion of minimal diffuse functions significantly improved the results for most functionals, but for BP86-D3BJ, MN15L, MN15, and M06, the use of larger aug-cc-pVTZ (or even ma-def2-TZVP for M06-D3) has no benefits for the accuracy. It might be explained by some error cancellation between geometry and single point energy, or some reasons concerning the fitting process when designing Minnesota's functionals.

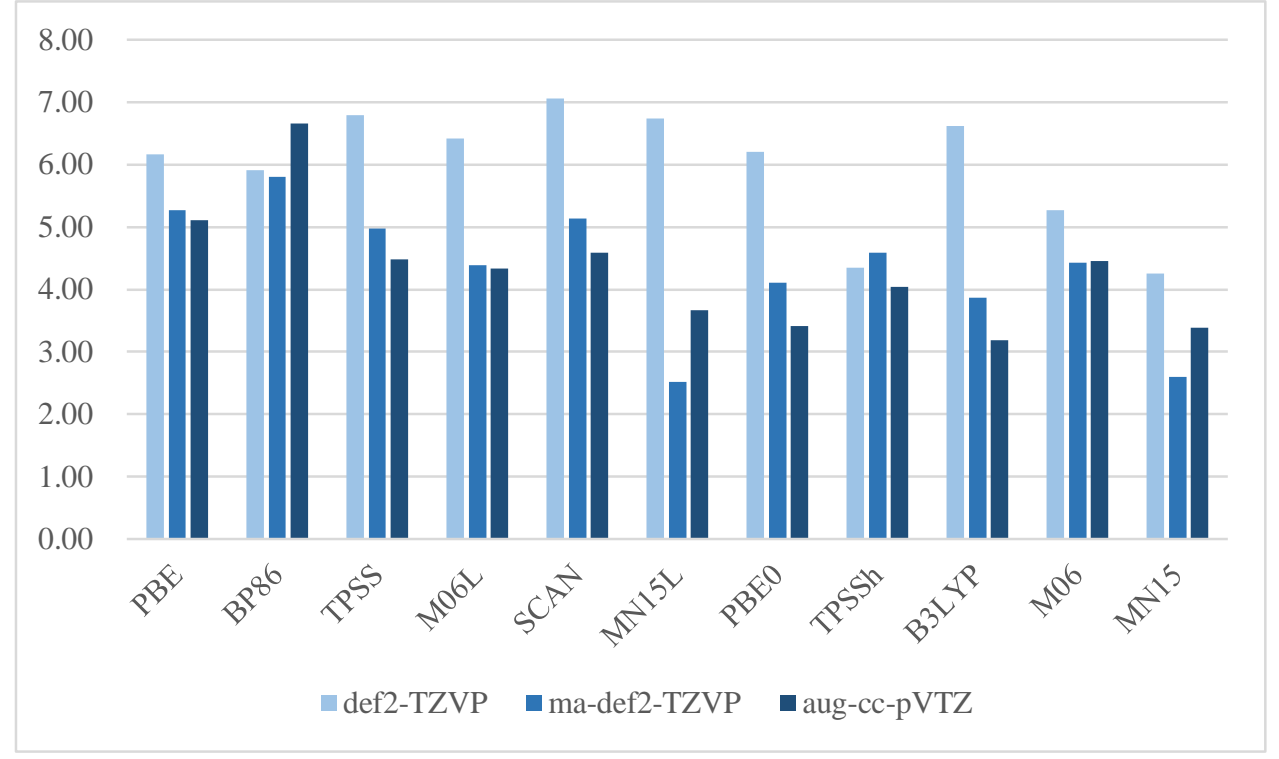

(a) 


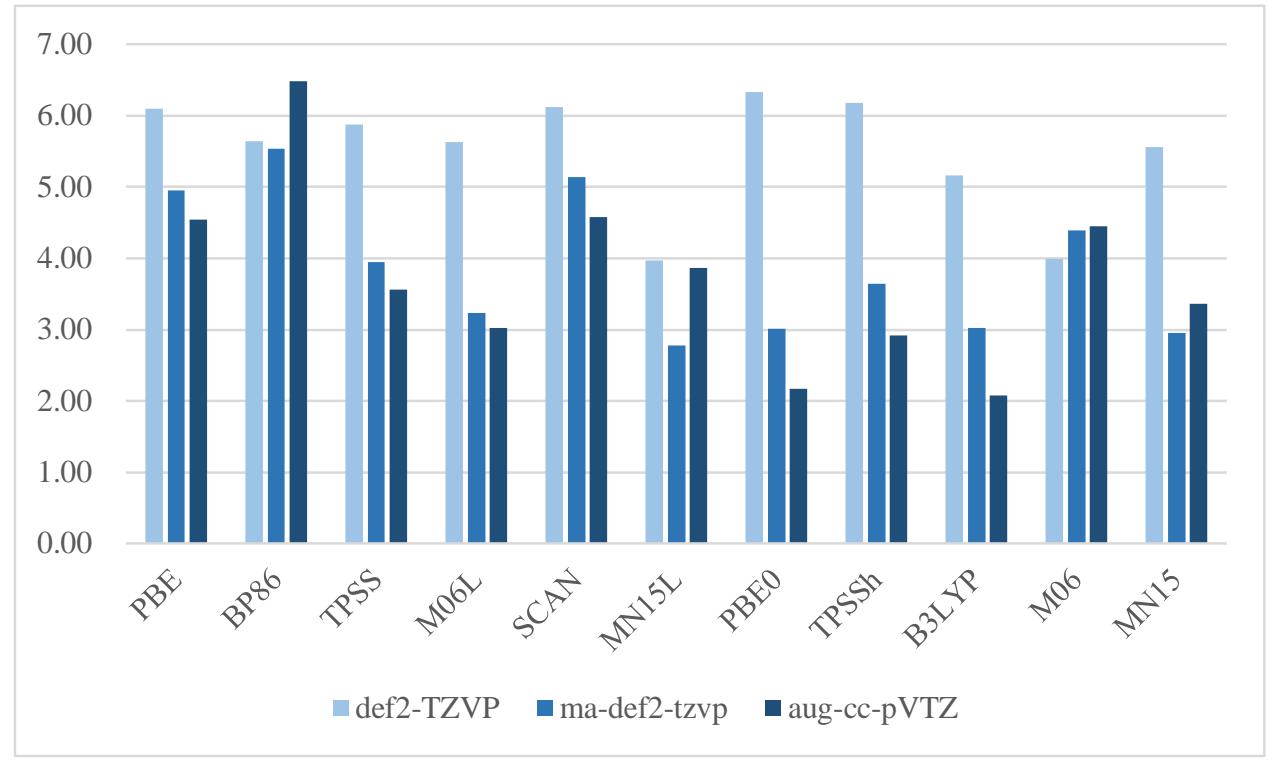

(b)

Figure 2. The MUEs (kcal/mol) for the overall testing set (a) and the monometallic set (b) of several functionals with increased basis set size.

\section{Higher-rank Functionals}

Some range-separated functionals and doubly-hybrid functionals were tested based on the MN15L/def2-TZVP structures, in combination with the aug-cc-pVTZ basis set, and the results are shown in Table 5. Astonishingly these higher-rank functionals gave much worse results. Since most of the doubly-hybrid functionals were fitted with 4-zeta basis set, some of the examples were reexamined at aug-cc-pVQZ level (Table 6). However, the improvement is negligible.

Although all the data were based on stable wavefunctions, it has to be noticed that in many cases, these functionals are very sensitive to initial guess for SCF procedure, and slightly different initial guess could lead to largely different results. Although similar phenomena also exist in other lower-rank functionals, the sensitivity towards initial guess is much more serious for doubly-hybrid functionals. Although much effort has been paid to find out the "correct" wavefunction, it cannot be concluded whether the failure of these functionals in this testing set is due to the functionals themselves, or because the wavefunction is not "correct".

Table 5. The performance of several range-separated and doubly-hybrid functionals (in $\mathrm{kcal} / \mathrm{mol}$ ). The reaction 3 was not included in MSE, MUE, Max Error and STDEV. 


\begin{tabular}{|c|c|c|c|c|c|c|c|c|}
\hline Reaction Number & wB97xD3 & wB97M-V & wB97X-V & B2PLYP & B2GPLYP & DSD-PBEP86 & wB2GPLYP & PWPB95 \\
\hline 1 & 10.68 & 17.18 & 10.31 & 10.16 & 7.11 & 7.34 & 14.86 & 19.58 \\
\hline 2 & -1.07 & 12.93 & 1.07 & -4.79 & 0.12 & -2.82 & 0.93 & -8.55 \\
\hline 3 & -44.88 & -42.18 & -44.28 & -18.50 & -3.91 & -35.29 & 20.90 & -49.75 \\
\hline 4 & 7.51 & 8.57 & 7.76 & 11.31 & 15.60 & 16.57 & 16.64 & 7.34 \\
\hline 5 & 4.08 & 8.76 & 4.52 & 0.51 & 6.39 & 5.84 & 5.94 & 5.25 \\
\hline 6 & -0.85 & -2.36 & 0.96 & 1.22 & 1.29 & -0.06 & 0.99 & 1.70 \\
\hline 7 & 4.83 & 8.49 & 6.30 & 3.17 & 1.03 & -1.19 & 1.67 & 3.99 \\
\hline 8 & -3.82 & -6.38 & 1.06 & 1.31 & 2.89 & 4.84 & 4.17 & 2.23 \\
\hline 9 & -3.89 & -5.51 & -0.30 & 5.36 & 9.58 & 9.57 & 9.37 & 4.77 \\
\hline 10 & -3.31 & -3.61 & 0.32 & -2.60 & 16.45 & 21.17 & 21.83 & -0.18 \\
\hline 11 & 0.68 & -1.55 & 3.64 & -1.58 & -9.69 & -13.79 & -10.85 & 2.59 \\
\hline 12 & -0.11 & -0.91 & 1.63 & 4.00 & 2.04 & 0.06 & -0.19 & 3.95 \\
\hline 13 & 1.37 & 1.27 & 7.17 & 2.14 & 3.92 & 4.03 & 5.83 & 3.65 \\
\hline 14 & 7.04 & 5.86 & 7.19 & 1.98 & -0.23 & -2.25 & -0.70 & 3.70 \\
\hline 15 & 3.83 & 1.45 & 3.02 & 1.23 & 0.80 & 1.03 & 0.79 & 2.31 \\
\hline 16 & -5.15 & -9.87 & 5.95 & -5.48 & -4.74 & -4.48 & -3.36 & -3.54 \\
\hline 17 & 6.60 & 5.93 & 8.00 & 1.57 & 11.38 & 17.20 & 13.09 & 5.80 \\
\hline 18 & 4.21 & 19.18 & 10.99 & 12.42 & 15.81 & 18.05 & 9.15 & 13.03 \\
\hline 19 & 9.16 & 8.52 & 10.62 & 8.23 & 7.14 & 5.40 & 5.12 & 8.40 \\
\hline MSE & 2.32 & 3.77 & 5.01 & 2.79 & 4.83 & 4.81 & 5.29 & 4.22 \\
\hline MUE & 4.34 & 7.13 & 5.05 & 4.39 & 6.46 & 7.54 & 6.97 & 5.59 \\
\hline Max Error & 10.68 & 19.18 & 10.99 & 12.42 & 16.45 & 21.17 & 21.83 & 19.58 \\
\hline STDEV & 4.68 & 7.93 & 3.64 & 4.98 & 6.90 & 8.83 & 7.67 & 5.80 \\
\hline
\end{tabular}

Table 6. Selected errors (in $\mathrm{kcal} / \mathrm{mol}$ ) for doubly-hybrid functionals with aug-cc-pVQZ basis set.

\begin{tabular}{|l|l|l|l|l|l|}
\hline $\begin{array}{l}\text { Reaction } \\
\text { Number }\end{array}$ & B2PLYP & B2GPPLYP & DSDPBEP86 & wB2GPPLYP & PWPB95 \\
\hline 7 & 3.11 & 0.91 & -1.15 & 1.67 & 3.97 \\
\hline 8 & 1.10 & 2.66 & 4.94 & 4.00 & 1.99 \\
\hline 9 & 6.23 & 6.94 & 8.92 & 7.42 & 6.07 \\
\hline 16 & -5.87 & -5.28 & -4.97 & -3.85 & -3.72 \\
\hline
\end{tabular}

\section{Conclusion}

Based on a testing set containing 19 open shell first-row transition metal reactions, a benchmark study for the accuracy of density functionals was performed. For the monometallic testing set, the PBE0-D3BJ and B3LYP-D3BJ exhibited the optimal performance. However, these two functionals failed to give acceptable results for bimetallic testing sets, whereas MN15 and MN15L exhibited balanced performance for monometallic and bimetallic examples. Although widely used, the GGA functionals PBE-D3BJ and BP86-D3BJ failed to afford as accurate results as those with higher rung in Jacob's ladder, even for the bimetallic testing set. On the other hand, the single-reference DLPNO-CCSD(T)/aug-cc-pVTZ is not suitable to be considered as the "gold standard" as compared to experimental values, and the results with higher-rank range-separated or doubly-hybrid functionals were unexpectedly bad. Although one cannot conclude whether it is due to the problems involving these doubly-hybrid functionals themselves, the results indicate that the use of doubly-hybrid functionals might be risky for compounds with complex electronic structures. Overall, the PBE0-D3BJ and B3LYP-D3BJ are highly recommended for the computational study of monometallic compounds, and MN15L for bimetallic complexes.

\section{References:}


1. Kohn, W., Nobel Lecture: Electronic structure of matter - wave functions and density functionals. Reviews of Modern Physics 1999, 71, (5), 1253.

2. Quintal, M. M.; Karton, A.; Iron, M. A.; Boese, A. D.; Martin, J. M., Benchmark study of DFT functionals for late-transition-metal reactions. The Journal of Physical Chemistry A 2006, 110, (2), 709-716 .

3. Weymuth, T.; Couzijn, E. P.; Chen, P.; Reiher, M., New benchmark set of transition-metal coordination reactions for the assessment of density functionals. Journal of chemical theory and computation 2014, 10, (8), 3092--3103.

4. Dohm, S.; Hansen, A.; Steinmetz, M.; Grimme, S.; Checinski, M. P., Comprehensive thermochemical benchmark set of realistic closed-shell metal organic reactions. Journal of chemical theory and computation 2018, 14, (5), 2596--2608.

5. Schultz, N. E.; Zhao, Y.; Truhlar, D. G., Density functionals for inorganometallic and organometallic chemistry. The Journal of Physical Chemistry A 2005, 109, (49), 11127--11143.

6. Zhao, Y.; Truhlar, D. G., Comparative assessment of density functional methods for $3 \mathrm{~d}$ transitionmetal chemistry. The Journal of chemical physics 2006, 124, (22), 224105.

7. Furche, F.; Perdew, J. P., The performance of semilocal and hybrid density functionals in $3 \mathrm{~d}$ transition-metal chemistry. The Journal of chemical physics 2006, 124, (4), 044103.

8. Peverati, R.; Truhlar, D. G., Quest for a universal density functional: the accuracy of density functionals across a broad spectrum of databases in chemistry and physics. Philosophical Transactions of the Royal Society A: Mathematical, Physical and Engineering Sciences 2014, 372, (2011), 20120476.

9. Schultz, N. E.; Zhao, Y.; Truhlar, D. G., Databases for transition element bonding: Metal- metal bond energies and bond lengths and their use to test hybrid, hybrid meta, and meta density functionals and generalized gradient approximations. The Journal of Physical Chemistry A 2005, 109, (19), 4388-4403.

10. Haoyu, S. Y.; He, X.; Li, S. L.; Truhlar, D. G., MN15: A Kohn--Sham global-hybrid exchange-correlation density functional with broad accuracy for multi-reference and single-reference systems and noncovalent interactions. Chemical science 2016, 7, (8), 5032--5051.

11. Jensen, K. P.; Roos, B. O. R. O.; Ryde, U., Performance of density functionals for first row transition metal systems. The Journal of chemical physics 2007, 126, (1), 014103.

12. Zheng, W.; Eustis, S. N.; Li, X.; Nilles, J. M.; Thomas, O. C.; Bowen, K. H.; Kandalam, A. K., Photoelectron spectroscopic study of iron--benzene cluster anions. Chemical Physics Letters 2008, 462, (1-3), 35--39.

13. Judai, K.; Hirano, M.; Kawamata, H.; Yabushita, S.; Nakajima, A.; Kaya, K., Formation of vanadium-arene complex anions and their photoelectron spectroscopy. Chemical physics letters 1997, 270, (1-2), 23--30.

14. Turner, N. J.; Martel, A. A.; Waller, I. M., Electron affinity of dicarbonylnitrosylcobalt (Co (CO) 2NO) measured by negative-ion photoelectron spectroscopy. The Journal of Physical Chemistry 1994, 98, (2), 474--477.

15. Stevens Miller, A. E.; Feigerle, C. S.; Lineberger, W. C., Laser photoelectron spectroscopy of CrH-, $\mathrm{CoH}-$, and NiH-: Periodic trends in the electronic structure of the transition-metal hydrides. The Journal of chemical physics 1987, 87, (3), 1549--1556.

16. Stevens, A. E.; Feigerle, C. S.; Lineberger, W. C., Laser photoelectron spectroscopy of MnH- and $\mathrm{FeH}-$ : Electronic structures of the metal hydrides, identification of a low-spin excited state of $\mathrm{MnH}$, and evidence for a low-spin ground state of FeH. The Journal of chemical physics 1983, 78, (9), 5420--5431. 
17. Miller, A. E.; Feigerle, C. S.; Lineberger, W. C., Laser photoelectron spectroscopy of MnH- 2, FeH2, $\mathrm{CoH}-2$, and NiH- 2: Determination of the electron affinities for the metal dihydrides. The Journal of chemical physics 1986, 84, (8), 4127--4131.

18. Andersen, T.; Lykke, K. R.; Neumark, D. M.; Lineberger, W. C., Autodetachment study of the electronic spectroscopy of FeO-. The Journal of chemical physics 1987, 86, (4), 1858--1867.

19. Zhang, N.; Hayase, T.; Kawamata, H.; Nakao, K.; Nakajima, A.; Kaya, K., Photoelectron spectroscopy of iron--sulfur cluster anions. The Journal of chemical physics 1996, 104, (10), 3413--3419. 20. Rathbone, G. J.; Sanford, T.; Andrews, D.; Lineberger, W. C., Photoelectron imaging spectroscopy of $\mathrm{Cu}-(\mathrm{H} 2 \mathrm{O})$ 1, 2 anion complexes. Chemical physics letters 2005, 401, (4-6), 570--574.

21. Capron, L.; Feng, W. Y.; Lifshitz, C.; Tjelta, B. L.; Armentrout, P. B., Reactions and thermochemistry of small cluster ions: $\mathrm{Fe}(\mathrm{CS} 2) \mathrm{n}+(\mathrm{n}=1,2)$. The Journal of Physical Chemistry 1996, 100, (41), 16571--16576.

22. Sievers, M. R.; Jarvis, L. M.; Armentrout, P. B., Transition-Metal Ethene Bonds: Thermochemistry of $\mathrm{M}+(\mathrm{C} 2 \mathrm{H} 4) \mathrm{n}(\mathrm{M}=\mathrm{Ti}-\mathrm{Cu}, \mathrm{n}=1$ and 2) Complexes. Journal of the American Chemical Society 1998, 120, (8), 1891--1899.

23. Casey, S. M.; Leopold, D. G., Negative ion photoelectron spectroscopy of $\mathrm{Cr} 2 \mathrm{H}$ and $\mathrm{Cr} 2 \mathrm{D}$. Chemical physics letters 1993, 201, (1-4), 205--211.

24. Zheng, W.; Nilles, J. M.; Thomas, O. C.; Bowen Jr, K. H., Photoelectron spectroscopy of nickelbenzene cluster anions. The Journal of chemical physics 2005, 122, (4), 044306.

25. Wu, H.; Desai, S. R.; Wang, L., Observation and Photoelectron Spectroscopic Study of Novel Mono-and Diiron Oxide Molecules: FeO y-(y= 1- 4) and Fe2O y-(y= 1- 5). Journal of the American Chemical Society 1996, 118, (22), 5296--5301.

26. Neese, F., The ORCA program system. Wiley Interdisciplinary Reviews: Computational Molecular Science 2012, 2, (1), 73--78.

27. Neese, F., Software update: the ORCA program system, version 4.0. Wiley Interdisciplinary Reviews: Computational Molecular Science 2018, 8, (1), e1327.

28. Perdew, J. P.; Burke, K.; Ernzerhof, M., Generalized gradient approximation made simple. Physical review letters 1996, 77, (18), 3865.

29. Grimme, S.; Antony, J.; Ehrlich, S.; Krieg, H., A consistent and accurate ab initio parametrization of density functional dispersion correction (DFT-D) for the 94 elements H-Pu. The Journal of chemical physics 2010, 132, (15), 154104.

30. Perdew, J. P., Phys. Re V. B 1986, 33, 8822. Erratum. Phys. Rev. B 1986, 34, 7406.

31. Becke, A. D., Density functional calculations of molecular bond energies. The Journal of Chemical Physics 1986, 84, (8), 4524--4529.

32. Tao, J.; Perdew, J. P.; Staroverov, V. N.; Scuseria, G. E., Climbing the density functional ladder: Nonempirical meta--generalized gradient approximation designed for molecules and solids. Physical Review Letters 2003, 91, (14), 146401.

33. Zhao, Y.; Truhlar, D. G., The M06 suite of density functionals for main group thermochemistry, thermochemical kinetics, noncovalent interactions, excited states, and transition elements: two new functionals and systematic testing of four M06-class functionals and 12 other functionals. Theoretical Chemistry Accounts 2008, 120, (1-3), 215--241.

34. Yu, H. S.; He, X.; Truhlar, D. G., MN15-L: A new local exchange-correlation functional for Kohn-Sham density functional theory with broad accuracy for atoms, molecules, and solids. Journal of chemical theory and computation 2016, 12, (3), 1280--1293. 
35. Staroverov, V. N.; Scuseria, G. E.; Tao, J.; Perdew, J. P., Comparative assessment of a new nonempirical density functional: Molecules and hydrogen-bonded complexes. The Journal of chemical physics 2003, 119, (23), 12129--12137.

36. Sun, J.; Ruzsinszky, A.; Perdew, J. P., Strongly constrained and appropriately normed semilocal density functional. Physical review letters 2015, 115, (3), 036402.

37. Stephens, P. J.; Devlin, F. J.; Chabalowski, C.; Frisch, M. J., Ab initio calculation of vibrational absorption and circular dichroism spectra using density functional force fields. The Journal of physical chemistry 1994, 98, (45), 11623--11627.

38. Adamo, C.; Barone, V., Toward reliable density functional methods without adjustable parameters: The PBE0 model. The Journal of chemical physics 1999, 110, (13), 6158--6170.

39. Chai, J.; Head-Gordon, M., Long-range corrected hybrid density functionals with damped atom-atom dispersion corrections. Physical Chemistry Chemical Physics 2008, 10, (44), 6615--6620.

40. Mardirossian, N.; Head-Gordon, M., \$lomega\$ B97M-V: A combinatorially optimized, rangeseparated hybrid, meta-GGA density functional with VV10 nonlocal correlation. The Journal of chemical physics 2016, 144, (21), 214110.

41. Mardirossian, N.; Head-Gordon, M., \$lomega\$B97X-V: A 10-parameter, range-separated hybrid, generalized gradient approximation density functional with nonlocal correlation, designed by a survivalof-the-fittest strategy. Physical Chemistry Chemical Physics 2014, 16, (21), 9904--9924.

42. Grimme, S., Semiempirical hybrid density functional with perturbative second-order correlation. The Journal of chemical physics 2006, 124, (3), 034108.

43. Karton, A.; Tarnopolsky, A.; Lam E Re, J.; Schatz, G. C.; Martin, J. M., Highly accurate firstprinciples benchmark data sets for the parametrization and validation of density functional and other approximate methods. Derivation of a robust, generally applicable, double-hybrid functional for thermochemistry and thermochemical kinetics. The Journal of Physical Chemistry A 2008, 112, (50), 12868--12886.

44. Kozuch, S.; Martin, J. M., DSD-PBEP86: in search of the best double-hybrid DFT with spincomponent scaled MP2 and dispersion corrections. Physical Chemistry Chemical Physics 2011, 13, (45), 20104--20107.

45. Casanova-P A Ez, M.; Dardis, M. B.; Goerigk, L., \$lomega\$B2PLYP and \$lomega\$B2GPPLYP: The First Two Double-Hybrid Density Functionals with Long-Range Correction Optimized for Excitation Energies. Journal of chemical theory and computation 2019, 15, (9), 4735--4744.

46. Grimme, S., Semiempirical hybrid density functional with perturbative second-order correlation. The Journal of chemical physics 2006, 124, (3), 034108.

47. Weigend, F.; Ahlrichs, R., Balanced basis sets of split valence, triple zeta valence and quadruple zeta valence quality for $\mathrm{H}$ to $\mathrm{Rn}$ : Design and assessment of accuracy. Physical Chemistry Chemical Physics 2005, 7, (18), 3297--3305.

48. Neese, F.; Wennmohs, F.; Hansen, A.; Becker, U., Efficient, approximate and parallel Hartree-Fock and hybrid DFT calculations. A 'chain-of-spheres' algorithm for the Hartree--Fock exchange. Chemical Physics 2009, 356, (1-3), 98--109.

49. Kendall, R. A.; Dunning Jr, T. H.; Harrison, R. J., Electron affinities of the first-row atoms revisited. Systematic basis sets and wave functions. The Journal of chemical physics 1992, 96, (9), 6796--6806.

50. Zheng, J.; Xu, X.; Truhlar, D. G., Minimally augmented Karlsruhe basis sets. Theoretical Chemistry Accounts 2011, 128, (3), 295--305.

51. Gorelsky, S. I., Complexes with a single metal--metal bond as a sensitive probe of quality of 
exchange-correlation functionals. Journal of chemical theory and computation 2012, 8, (3), 908--914. 52. Bauschlicher Jr, C. W.; Ram, R. S.; Bernath, P. F.; Parsons, C. G.; Galehouse, D., The A 6 \$ISigma\$+-X 6 \$ Sigma \$+ transition of CrH, Einstein coefficients, and an improved description of the A state. The Journal of Chemical Physics 2001, 115, (3), 1312--1318. 